\title{
El gestor bibliográfico digital colaborativo como herramienta de apoyo al proceso de investigación
}

\author{
William-Enrique Mercado-Borja, Juancri-Rusbel Barrera-Navarro y Roisman-Enrique
}

Ravelo-Méndez

\section{RESUMEN}

Este es un estudio de caso con enfoque cuali-cuantitativo y nivel descriptivo, sobre cómo los estudiantes perciben el uso del gestor bibliográfico colaborativo digital. El estudio ayuda a reconocer la relevancia de utilizar dicho gestor para cualificar la investigación y la producción académica, desde proyectos que desarrollan estudiantes de la maestría en Tecnologías Digitales Aplicadas a la Educación. Participaron 103 estudiantes de los módulos: Caracterización de la investigación aplicada y Propuesta de investigación aplicada. Los resultados dejan ver que los tesistas valoran positivamente el gestor como dispositivo tecnológico clave para la gestión de la información y del conocimiento; a su vez, permiten inferir que esta herramienta informática en mención facilita la búsqueda, clasificación y análisis de registros bibliográficos, traduciéndose en adecuadas toma de decisiones y mejora en el desempeño tanto académico como de investigación.

Palabras clave: conocimiento, gestor bibliográfico, interactividad virtual, investigación aplicada, tecnología educacional, Colombia.

William-Enrique Mercado-Borja

xwimer@yahoo.com

Colombiano. Licenciado en Informática Educativa y Medios Audiovisuales, Universidad de Córdoba, Colombia. Docente investigador del grupo de investigación BIMADINo de la Universidad de Córdoba, Colombia. Temas de investigación: interactividad virtual, interacción socio técnica y acciones innovadoras mediadas con TIC. ORCID: https://orcid.org/0000-0003-4872-0130.

\section{Juancri-Rusbel Barrera-Navarro}

\section{Roisman-Enrique Ravelo-Méndez}

roisman.ravelo@cvudes.edu.co

Colombiano. Licenciado en educación básica con énfasis en tecnología e informática, Corporación Universitaria de Caribe (CECAR), Colombia. Docente de posgrado de la Universidad de Santander, Colombia; miembro del grupo de investigación Veritas AID, Colombia. Temas de investigación: educación virtual, pedagogía y TIC, innovación de prácticas pedagógicas. ORCID: https://orcid. org/0000-0002-1028-8859. 


\section{O gestor bibliográfico digital colaborativo como ferramenta de apoio ao processo de investigação}

\section{RESUMO}

Este é um estudo de caso com enfoque qualitativo-quantitativo e nível descritivo, sobre como os estudantes percebem o uso do gestor bibliográfico colaborativo digital. O estudo ajuda a reconhecer a relevância de utilizar dito gestor para qualificar a pesquisa e a produção acadêmica, desde projetos que desenvolvem estudantes do mestrado em Tecnologias Digitais Aplicadas à Educação. Participaram 103 estudantes dos módulos: Caracterização da investigação aplicada e Proposta de investigação aplicada. Os resultados mostram que os alunos valorizam positivamente o gestor como dispositivo tecnológico chave para a gestão da informação e do conhecimento; por sua vez, permitem inferir que esta ferramenta informática facilita a busca, classificação e análise de registros bibliográficos, traduzindo-se em adequadas toma de decisões e melhora no desempenho tanto acadêmico como de investigação.

Palavras chave: conhecimento, gestor bibliográfico, interatividade virtual, investigação aplicada, tecnologia educacional, Colômbia.

\section{The collaborative digital bibliographic manager as a tool to support the research process}

\section{ABSTRACT}

This is a case study with both a qualitative and quantitative approach and a descriptive level about how students perceive the use of the collaborative digital bibliographic manager. The study helps to acknowledge the relevance of using this manager to qualify research and academic production, from projects developed by students of the master's degree in Digital Technologies Applied to Education. A total of 103 students took part in the units Characterization of applied research and Applied research proposal. The results show that the graduates value positively the manager as a key technological device for information and knowledge management; at the same time, they infer that this computer tool facilitates the search, classification and analysis of bibliographic records, resulting in adequate decision making and improvement in both academic and research performance.

Key words: knowledge, bibliographic manager, virtual interactivity, applied research, educational technology, Colombia. 


\section{Introducción}

Uno de los principales propósitos en programas de maestría es que el estudiante construya conocimientos o genere aportes de valor que sirvan para el enriquecimiento académico y la comprensión de un objeto de estudio. En este trabajo se argumenta que es importante que los estudiantes comprendan la funcionalidad de un gestor bibliográfico colaborativo digital (GBCD) y los contenidos que el mismo posee, pues éste será una herramienta muy útil en su trabajo de investigación. Este trabajo implica el desarrollo de conceptualizaciones y competencias que, de una forma u otra, ayudan a dar respuestas a necesidades sociales o tecnológicas concretas, por ello, se debe garantizar el acceso, uso y apropiación de las tecnologías de la información y la comunicación (TIC), que ejerzan un papel inclusivo, participativo e innovador, como medio para optimizar la calidad educativa y de paso incrementar la implicación del estudiantado en sus tareas, ya que se ven obligados constantemente a tomar decisiones, a filtrar, a escoger y seleccionar información (Bautista, 2007).

En consecuencia, las tecnologías digitales educacionales se tornan indispensables en diversos tipos de formación, dado que se convierten en mecanismos de apoyo pedagógico, posibilitan el trabajo interactivo, auspician la interacción social y permiten la gestión de la información y del conocimiento. Por lo antedicho, se buscó identificar los usos del gestor bibliográfico colaborativo digital como apoyo al desarrollo de actividades académicas y de investigación en red, puesto que hay pocos estudios que dejan ver la importancia de las buenas prácticas educativas con el gestor en mención.

Es necesario destacar que la sociedad del conocimiento lleva a que toda unidad de trabajo deba documentarse respecto al tema de estudio y de paso organizar la información requerida para desarrollar a feliz término las distintas fases o etapas que hacen parte de un proyecto de investigación. De ahí que para la generación de producciones académicas, "es necesario gestionar información y desarrollar una profunda revisión, definir la calidad de búsqueda obtenida, hacer un adecuado uso de las citas, junto con la inclusión de las referencias bibliográficas" (Reyes, Cárdenas y Aguirre, 2020a: 233).

El estudio contribuye al análisis de la implantación de una didáctica apoyada en el uso del GBCD para respaldar aprendizajes y procesos investigativos desde la virtualidad. Esto es importante "porque favorece al cambio de paradigma de los docentes con relación a la utilización de las TIC en sus prácticas pedagógicas cotidianas como una estrategia capaz de desarrollar competencias básicas y tecnológicas" (Cárdenas et al., 2019: 76). El estudio tiene también el propósito de conocer que tan útil es el GBCD para la gestión y organización de registros bibliográficos. De ahí que se planteó la pregunta ¿cómo influye el gestor bibliográfico colaborativo digital en el proceso formativo e investigativo de estudiantes de la maestría en Tecnologías Digitales Aplicadas a la Educación?

Finalmente, el artículo se inscribe en una indagación con la intención de conocer, analizar y valorar el uso del GBCD y su influencia en los módulos de Caracterización de la investigación aplicada y Propuesta de investigación aplicada (conjunto de módulos obligatorios, en la línea de desarrollo del trabajo de grado de la maestría mencionada), desde una perspectiva pedagógica, tecnológica e investigativa. Lo anterior, debe ser considerado por los diversos actores del proceso educativo, a fin y efecto de garantizar y asegurar la eficiencia práctica de la gestión tecnológica y del conocimiento.

\section{El concepto de gestor bibliográfico y sus implicaciones en la investigación}

El término gestor bibliográfico es utilizado desde diferentes escenarios, tales como: el tecnológico, informático, educativo, investigativo, entre otros. Se realizó un análisis desde distintas definiciones que dejan ver la diversidad de las bases teóricas de éste; 
el gestor bibliográfico es un objeto de discusión que ha propiciado múltiples definiciones y perspectivas a lo largo del tiempo.

Cabe señalar que los softwares de gestión de referencia han sido una herramienta útil para los investigadores desde la década de 1980. En esos primeros años, se hicieron ad hoc, y algunos se basaron en el sistema de gestión de base de datos dBase II/III (Bertrand y Bader, 1980; Kunin, 1985). Por un lado, se estaría hablando de aplicaciones con fines personales y notable tradicionalidad respecto a la gestión de la información; por otro, surgen definiciones conceptuales pasivas por la naturaleza misma del artefacto tecnológico en su momento. De hecho, "la literatura consigna que los primeros fueron, ProCite y Reference Manager, creados en 1983 y 1984. Posteriormente en la década de 1990, el hecho de que se extendiera el acceso a bases de datos bibliográficas desde Internet" (Reyes, Cárdenas y Aguirre, 2020b: 233).

Durante la década del 2000 la expresión gestor bibliográfico cobró mayor fuerza, gracias a la llegada de la web 2.0 y a la creación de nuevos gestores en aras de materializar los beneficios ofrecidos por dicha llegada. Por ello, los software Zotero y Mendeley, terminan siendo de interés para algunos académicos, teóricos, investigadores y profesionales. Más tarde, se utiliza el término gestor bibliográfico colaborativo, el cual dio lugar a un nuevo matiz conceptual que involucraría principios del trabajo colaborativo y de los servicios, o distintas opciones de interactividad que inicio ofreciendo la web 3.0.

Desde el aspecto académico, Martínez et al. (2013: 3) consideran que el gestor bibliográfico "es un software para estudiantes e investigadores cuya utilidad es guardar, modificar y usar citas bibliográficas de determinados documentos", o sea que la utilidad académica y la diversidad de fuentes terminan siendo el eje central del artefacto en mención, sin importar el recurso tecnológico usado para ello. Armenteros y Alfonso (2004, citado por Duarte, 2007: 647 interpretan la herramienta desde lo particular, conceptualizándola como "gestores personales de bases de datos de referencias bibliográficas, que son programas que permiten a investigadores, especialistas y profesionales almacenar las referencias bibliográficas obtenidas durante la búsqueda documental"; por tanto el usuario final puede gestionar el contenido, es decir, la tecnología se convierte en un medio que potencia la información necesaria para la construcción de nuevos conocimientos y al cumplimiento de normas técnicas o científicas. Después de todo, ambas perspectivas tienen un punto en común, y es que sitúan al actor en el centro de la actividad informática, por gestionar la información.

Generalmente, el concepto de gestor bibliográfico se asocia a fuentes de información, principalmente con referencias bibliográficas, dado que se ha convertido en un mecanismo que posibilita la actualización, organización y el almacenamiento de contenidos mediante tecnologías de información. De ahí que Fernández, Goicochea, Hernández y López (2012) refieren al gestor bibliográfico como un mecanismo informático que sirve para "citar e incluir automáticamente las fuentes consultadas en los trabajos, libros, artículos, según las normas de cita requeridas", permitiendo que el actor educativo comprenda y transforme sus ideas o fases, como parte del proceso de investigación. Por ello, es muy importante que el uso y apropiación de este tipo de tecnologías impacten positivamente y se conviertan en medios que potencien el $e$-Learning y el saber.

Por lo anterior, internet se convierte en un escenario que propicia la cualificación y construcción conjunta de trabajos de indagación desde el interés y la participación colaborativa de los futuros magister. En consecuencia, plantear procesos que lleven al diseño y desarrollo de estudios se vuelve un poco complejo, ya que ameritan la identificación de aspectos y acciones que ayuden a cualificar la producción académica y lleven al avance científico apoyados con TIG, y a la empleabilidad de espacios de socialización para fortalecer las prácticas de investigación. Por tanto, se 
puede decir, que el "proceso de mejora es multi-causal y constituye sin duda un esfuerzo colectivo de los diferentes actores involucrados" (Solórzano, García, Lesser, Koller y Ward, 2016: 9).

La "gran red" brinda herramientas tecnológicas para que los estudiantes puedan "generar, gestionar y apropiarse de la información y el conocimiento" (Bianco, Lugones y Peirano, 2016: 16), al igual que a desarrollar procesos colaborativos, más aún cuando la interactividad "tiende un puente entre los medios y los lectores o usuarios, porque permite abordar ese espacio de relación entre ambas partes" (Rost, 2014, citado por Méndez, Peñaranda y Rojas, 2018: 36); lo cua permite comprender los procesos de interactividad dados en el GBCD y de paso mejorar su operatividad en aras de aumentar la calidad académica e investigativa.

Para Ramdhani et al. (2014, citado por Rodríguez, 2020: 17), los gestores bibliográficos permiten la revisión de fuentes fiables y de interés que ayudan a dilucidar "el panorama de investigación mediante una visión general de fuentes exploradas, lo cual afianza el trabajo científico o académico e indica a los lectores como el proyecto de investigación se ajusta a un campo general de estudio más amplio". En consecuencia, se puede decir que en cierta medida existe una acción recíproca entre el investigador y la fuente bibliográfica y que puede terminar en un intercambio de papeles; es decir, que no siempre el actor desde su accionar cuestiona el contenido, sino que el contenido puede cuestionar la perspectiva de pensamiento del investigador en cualquier escenario social, tecnológico o educativo.

Por consiguiente, es claro que los procesos de investigación aplicada "demandan de voluntades y responsabilidades individuales, colectivas e institucionales que deben converger en un interés común y llevar a una cultura organizativa" (Mercado, Guarnieri y Rodríguez, 2019: 72). De modo que la investigación requiere del compromiso y la participación proactiva de los actores involucrados tanto en el proceso formativo como en el investigativo, a fin y efecto de alcanzar logros científicos, sociales y tecnológicos en función de la calidad educativa.

Desde otra perspectiva, Fernández et al. (2012) proponen que el gestor bibliográfico sirve para "citar e incluir automáticamente fuentes consultadas en trabajos, libros, artículos, según las normas de cita requeridas". De ahí que la universidad debe poner a disposición de estudiantes y docentes algunos gestores bibliográficos fáciles de localizar. Así, los estudiantes deben tener la posibilidad de modificar la configuración del gestor para aumentar el nivel de interactividad; así el actor del proceso educacional tiene la oportunidad de recopilar, organizar o editar documentos o contenidos. Esto permitiría encauzar propósitos y responsabilidades a medida que se desarrollan los módulos Caracterización de la investigación aplicada y Propuesta de investigación aplicada.

De igual modo, el GBCD no sólo debe generar un intercambio, sino también procesos de "edición bidireccional y multidireccional de mensajes y objetos en un marco de trabajo colaborativo, abierto, democrático y plural. De modo que no se puede pensar la interactividad separada de las posibilidades de intercambiar, modificar y transmitir mensajes y objetos online" (Guarnieri, 2018: 102). El gestor bibliográfico como tecnología de información también debe contar con mecanismos que estimulen la intersubjetividad y ayuden a fijar prioridades en una red de aprendizaje colaborativo, para auspiciar el proceso educativo e investigativo en un escenario mediado con infraestructura tecnológica; y más cuando la web 3.0 permite avanzar en el tema de innovación tecnológica e incorporación de nuevos conceptos, en lo que respecta a procesos de importación y exportación de comentarios, citas y referencias de manera multidireccional, construir o rediseñar objetos bibliográficos en formatos de valor, al igual que compartir las referencias bibliográficas.

En ese sentido, la "toma de decisiones permite que un problema o situación sea valorado para 
poder elegir la mejor alternativa" (Casco, Álvarez y Garrido, 2019: 55), por ello, al momento de realizar procesos de cualificación en un gestor bibliográfico colaborativo digital, es necesario que los actores educativos tengan nociones sobre gestión tecnológica y del conocimiento, para que participen con libertad y fundamento en toma de decisiones que conduzcan al logro de metas significativas. Cabe resaltar que pese a las mejoras que han tenido los gestores bibliográficos, éstos continúan siendo inseparables de su propio diseño conceptual operativo, puesto que por naturaleza existe una complementariedad entre sí que posibilita la reciprocidad entre lo tecnológico y lo social. En efecto, el GBCD requiere de una reconfiguración en pro de mejorar su intencionalidad para abordar situaciones más específicas, potenciar la gestión del conocimiento tanto educativo como investigativo, generar sentido de apropiación y, en última instancia, propiciar mecanismos que significativamente tributen al trabajo colaborativo en red; ahora bien, el "trabajo colaborativo en red es sólo una de las estrategias posibles para procesos de innovación social, marca la pauta para forjar las bases de la gestión del conocimiento" (Rincón, Hermith y Bautista, 2018: 56).

Finalmente, puede apreciarse la definición conceptual y operativa de lo que es el gestor bibliográfico y lo que ha significado para el desarrollo de procesos académicos y la investigación aplicada. Asimismo, se argumentó la relevancia e importancia de la herramienta tecnológica, ya que ha teniendo una significativa evolución en el ámbito educativo, tecnológico y científico. Sin duda alguna, los actuales enfoques, tipos y diseños de investigación aplicada propician nuevos escenarios para la educación virtual y la gestión del conocimiento.

\section{Metodología}

Este estudio se desarrolló desde un enfoque cualicuantitativo, puesto que se empleó una encuesta que permitió describir la situación actual del GBCD empleado en una comunidad de aprendizaje, al igual que interpretar la participación de la población objeto de estudio; por ello, se hizo un análisis de documentos relacionados con acciones innovadoras mediadas con TIC para el procesamiento y la búsqueda de información dentro del estudio; se empleó un cuestionario con escala tipo Likert. Las respuestas se tabularon y analizaron a partir de Microsoft Excel y de estadística descriptiva. Conviene anotar que, por un lado, la investigación cualitativa refiere a una "actividad sistemática orientada a la comprensión en profundidad de fenómenos educativos y sociales, a la transformación de prácticas y escenarios socioeducativos, a la toma de decisiones y también hacia el descubrimiento y desarrollo de un cuerpo organizado de conocimientos" (Sandín, 2003: 123); por otro lado, la investigación cuantitativa es "entendida como aquella que comprende procedimientos de recolección de datos, en la que se presentan datos numéricos que son analizados generalmente por métodos estadísticos" (Martínez, 2021: 39).

Asimismo, se indaga sobre las experiencias de los individuos en su contexto real y no busca manipular el entorno de la investigación (Mayan, 2001, citado por Guacaneme, 2014: 54). Con el fin de abordar y entender el ejercicio educativo respecto al uso del gestor bibliográfico colaborativo digital, se realiza un estudio de caso, enmarcado como descriptivo con diseño no experimental, ya que el investigador no manipulo las variables a medida que registraba la información. En cuanto al tiempo, se puede decir que es transversal, dado que los datos fueron recopilados una sola vez.

En la indagación participaron 103 estudiantes de los módulos: Caracterización de la investigación aplicada y Propuesta de investigación aplicada, de la maestría Tecnologías Digitales Aplicadas a la Educación (TDAE) de la Universidad de Santander, Colombia; se seleccionaron de manera intencional y voluntariamente respondieron el cuestionario empleado para apreciar y analizar sus opiniones. El 
instrumento fue validado por expertos en educación, piloteado e implementado a través de un formulario en línea, para luego tabular y analizar la información recopilada. La encuesta se aplicó con el objetivo de indagar sobre los beneficios y problemáticas halladas con el gestor bibliográfico colaborativo digital en el proceso formativo de los módulos mencionados.

En la tabla 1 se precisan con detalle las variables, técnica e indicadores que ayudan a materializar el objetivo de la investigación.

Tabla 1. Operación de los constructos

\begin{tabular}{|c|c|c|c|}
\hline Variable & Tipo de variable & Instrumentos-técnicas & Indicadores \\
\hline $\begin{array}{l}\text { Usos del gestor } \\
\text { bibliográfico } \\
\text { colaborativo digital }\end{array}$ & $\begin{array}{l}\text { Variable } \\
\text { cualitativa } \\
\text { politómica con } \\
\text { escala nominal }\end{array}$ & $\begin{array}{l}\text { - Cuestionario de } \\
\text { encuesta aplicada } \\
\text { a estudiantes de } \\
\text { maestría en Tecnologías } \\
\text { Digitales Aplicadas a la } \\
\text { Educación. } \\
\text { Técnicas estadísticas: } \\
\text { descripción: gráficas } \\
\text { de barra; análisis } \\
\text { unidimensional: tabla de } \\
\text { frecuencias }\end{array}$ & 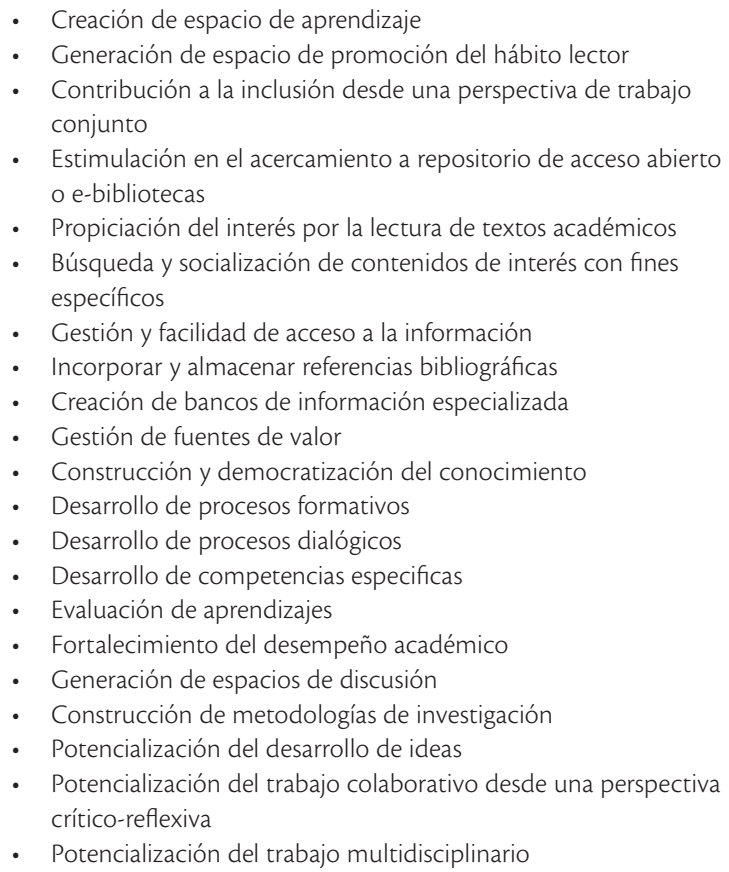 \\
\hline $\begin{array}{l}\text { Satisfacción } \\
\text { del maestrante } \\
\text { con el gestor } \\
\text { bibliográfico }\end{array}$ & $\begin{array}{l}\text { Variable } \\
\text { cualitativa } \\
\text { politómica con } \\
\text { escala ordinal }\end{array}$ & $\begin{array}{l}\text { - Cuestionario de } \\
\text { encuesta aplicada a } \\
\text { estudiantes de maestría } \\
\text { en TDAE. } \\
\text { Técnicas estadísticas: } \\
\text { descripción: gráficas } \\
\text { de barra; análisis } \\
\text { unidimensional: tabla de } \\
\text { frecuencias }\end{array}$ & $\begin{array}{l}\text { - Importancia del gestor bibliográfico en el proceso formativo e } \\
\text { - } \text { Resultados de investigación con ayuda del gestor bibliográfico } \\
\text { - } \text { Feedback investigativo o evaluativo mediante el uso del gestor } \\
\text { bibliográfico } \\
\text { - Resolución de dudas académicas o investigativas con el uso del } \\
\text { - } \text { gestor bibliográfico } \\
\text { - } \text { - Identiación de las características del pensamiento } \\
\text { gestor bibliográn del estado del arte de un objeto de estudio con el } \\
\text { - Trabajo autónomo propiciado por el gestor bibliográfico } \\
\text { colaborativo } \\
\text { - Trabajo colaborativo facilitado en gran medida por el gestor } \\
\text { bibliográfico } \\
\text { - Integración de conceptos de diversas áreas del saber mediante } \\
\text { - el gestor } \\
\text { Relación del gestor bibliográfico con la aplicación de conceptos } \\
\text { prácticos de interés }\end{array}$ \\
\hline
\end{tabular}

Fuente: elaboración propia. 


\section{Discusión y análisis de resultados}

El gestor bibliográfico colaborativo digital

\section{en la investigación aplicada}

La investigación aplicada permite conocer las situaciones que se dan en un objeto de estudio y relacionar aspectos técnicos, académicos y disciplinares asociados a la implementación del GBCD. En el estudio se consideraron indicadores relacionados con la aplicabilidad concebida por dicho gestor. En la tabla 1 se puede apreciar que los estudiantes de la maestría, para respaldar su proceso formativo y de investigación, se apoyan en esta herramienta informática, lo cual permite afirmar la importancia de ésta en el aprendizaje autónomo y en el desarrollo de módulos adscritos al área de investigación.

Tabla 2. Diversidad de usos del gestor bibliográfico colaborativo digital

\begin{tabular}{|c|c|c|}
\hline Indicador & Frecuencia absoluta & Frecuencia relativa $\%$ \\
\hline Crear espacio de aprendizaje & 43 & 41.7 \\
\hline Generar espacio de promoción del hábito lector & 8 & 7.8 \\
\hline Contribuir a la inclusión desde una perspectiva de trabajo conjunto & 12 & 11.7 \\
\hline Estimular el acercamiento a repositorios de acceso abierto o bibliotecas virtuales & 15 & 14.6 \\
\hline Propiciar interés por la lectura de textos académicos & 13 & 12.6 \\
\hline Buscar y socializar contenidos de interés con fines específicos & 49 & 47.6 \\
\hline Gestionar y facilitar el acceso a la información & 33 & 32.0 \\
\hline Incorporar y almacenar referencias bibliográficas & 42 & 40.8 \\
\hline Crear bancos de información especializada & 20 & 19.4 \\
\hline Gestionar fuentes de valor & 20 & 19.4 \\
\hline Construir y democratizar el conocimiento & 10 & 9.7 \\
\hline Desarrollar procesos formativos & 28 & 27.2 \\
\hline Desarrollar procesos dialógicos & 10 & 9.7 \\
\hline Desarrollar competencias específicas & 10 & 9.7 \\
\hline Evaluar aprendizajes & 4 & 3.9 \\
\hline Fortalecer el desempeño académico & 30 & 29.1 \\
\hline Generar espacio de discusión & 24 & 23.3 \\
\hline Construir metodologías de investigación & 19 & 18.4 \\
\hline Potenciar el desarrollo de ideas & 26 & 25.2 \\
\hline Potenciar el trabajo colaborativo desde una perspectiva crítico-reflexiva & 25 & 24.3 \\
\hline Potenciar el trabajo multidisciplinario & 12 & 11.7 \\
\hline
\end{tabular}




\section{Gráfica 1. Diversidad de usos del GBCD}

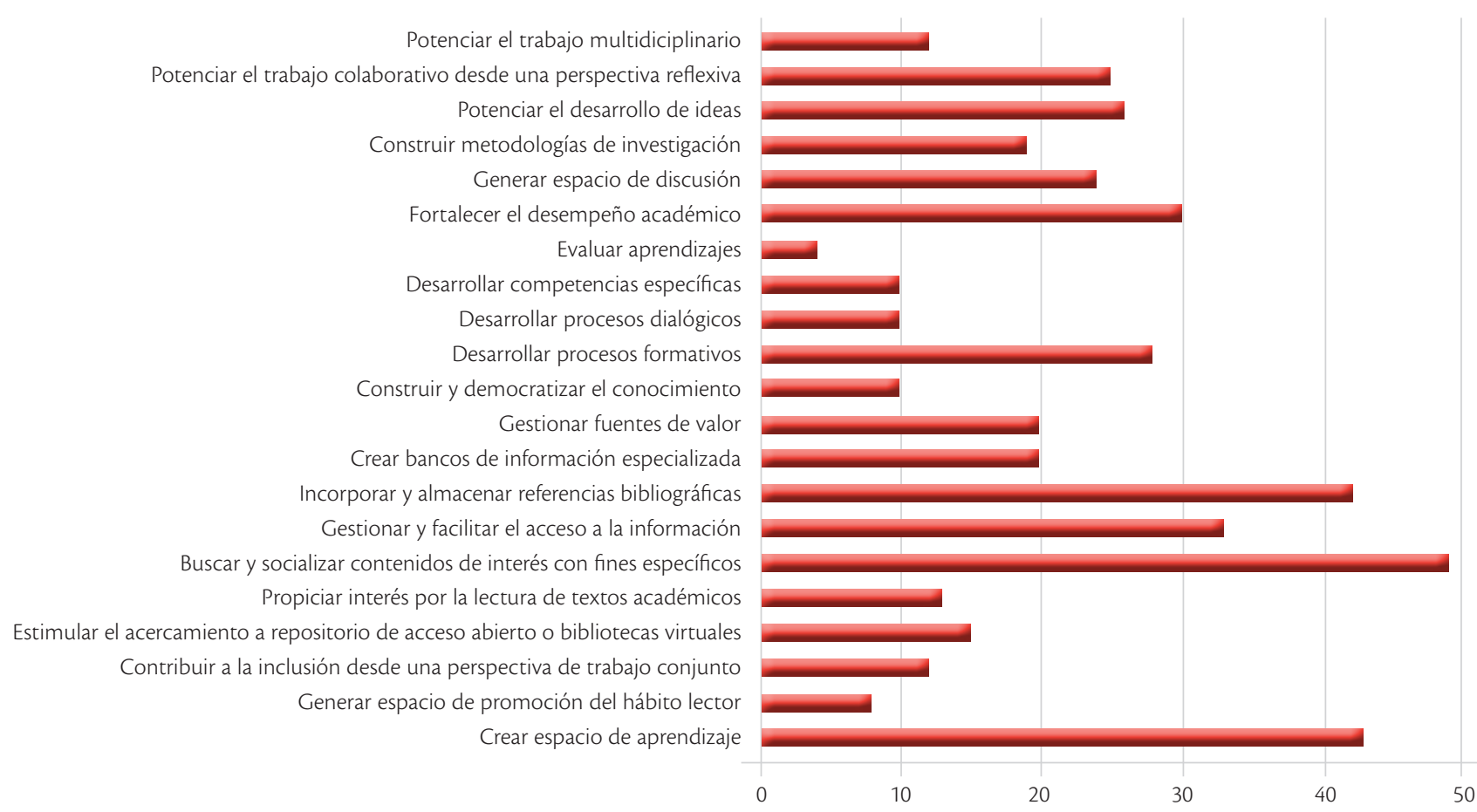

Fuente: elaboración propia.

En la tabla 2 (y en la gráfica 1) se puede observar que, para potenciar el proceso de investigación, los maestrantes utilizan significativamente el GBCD. Por ello, se convierte en un factor tecno-didáctico que apoya la praxis académica y, de paso, motiva a los estudiantes a que se apropien y utilicen ese medio a partir de sus motivaciones, necesidades e intereses. También se reconoce que el $80.95 \%$ de los indicadores abordados develan que el GBCD que usan los educandos en su ejercicio académico investigativo es una estrategia para integrar y administrar la información, impulsar la autonomía y respaldar el trabajo interactivo. De igual forma, los indicadores que se exponen en la tabla muestran que la acción investigativa del estudiante se centra en adquirir y almacenar la información.

Dentro del conjunto de datos, se observa que el $47.6 \%$ de los estudiantes se apoya en la búsqueda y socialización de contenidos para propiciar la disertación alrededor del pensamiento computacional con fundamentos teórico-prácticos ampliamente aceptados; el 40.8\% incorpora y almacena referencias bibliográficas con el fin de contribuir al desarrollo de competencias tecnológicas y de contenido. Además, el $41.7 \%$ cree que el GBCD se convierte en un espacio de aprendizaje; el 32\% se apoya en el gestor para facilitar el acceso, uso y apropiación de la información, y el $24.3 \%$ se apoya en esta tecnología con la intención de favorecer la formación extracurricular a partir del trabajo colaborativo desde una perspectiva crítico-reflexiva. Igualmente, un 29.1\% de los maestrantes cuenta con un buen nivel para fortalecer el desempeño académico mediante el uso del artefacto tecnológico en mención, un $27.2 \%$ considera que promueve el desarrollo de procesos formativos y un $25.2 \%$ manifiesta que contribuye al desarrollo de ideas, las cuales pueden ser socializadas para que sean valoradas, ampliadas y estimadas en justa medida por parte de los partícipes. 
Por lo antes expuesto, se plantea que la cultura organizacional y digital de la investigación aplicada sea realmente práctica, integral y multidisciplinar, pues "esta multidisciplinariedad deriva la generación de 'meta-interpretaciones' que prestan atención a los aspectos políticos, sociales y económicos de un fenómeno" (Olsen, 2004, citado por Rodríguez, 2005 : 36), para apostarle significativamente a la gestión del conocimiento educativo y al progreso tecnológico. En el análisis y la interpretación de los resultados respecto a la utilización del GBCD en procesos de investigación, se percibe la necesidad de democratizar el conocimiento y el trabajo multidisciplinar desde una apropiación integral del gestor y una triangulación interdimensional que garantice la calidad educativa y el avance científico; ello con el fin de adquirir y materializar en la investigación aplicada, las diversas miradas de los distintos actores educativos.

En consecuencia, la construcción de soluciones a través de la investigación aplicada y recursos tecnológicos de la web 3.0 "permite un nuevo nivel de integración de datos y aplicación inter operable" (Fernández, 2013) y a su vez, "el usuario tiene el control para hacer los cambios que desee modificando directamente las bases de datos" (Larrote, 2018: 5); además de ello, fortalece el proceso formativo y el perfil profesional de los estudiantes de la maestría TDAE. Así, "la tecnología incorporada a los procesos educativos puede ser un elemento enriquecedor y desarrollador para el aprendizaje" (Góngora y Martínez, 2012: 342), al igual que la investigación como estrategia integradora en el desarrollo de contenidos y la formación universitaria. En consecuencia, los datos recolectados dejan ver que el GBCD responde a necesidades y requerimientos comunes por parte de los encuestados, en lo que refiere al logro de competencias tecnológicas, investigativas y disciplinares.

Cabe señalar que, en el proceso de investigación, las TIC respaldan significativamente a los estudiantes de la maestría en mención, al proporcionarles información actualizada y fiable para la comprensión de diferentes objetos de estudio y un mejor beneficio académico; "las tecnologías pasan a desempeñar un papel preponderante, en tanto aseguran la provisión de información actualizada" (Litwin, 2005: 6).

\section{El gestor bibliográfico en la creación de propuestas de investigación aplicada}

La implementación del GBCD como herramienta de apoyo a las clases virtuales y tareas de los módulos fue valorada positivamente por los estudiantes. Además de ser una herramienta informativa no obligatoria dentro de dichos módulos a través de actividades complementarias, tuvo un alto uso, develando que los encuestados tienen una buena percepción del gestor, al considerar que ayuda a cualificar el e-Learning y el desempeño académico.

En relación con la posibilidad de trabajo colaborativo, en la tabla 3 (gráfica 2) se observa que, el $89.32 \%$ cree que el gestor lleva a ello; el $88.34 \%$ señala que el feedback investigativo o evaluativo propiciado por la herramienta auspicia el proceso de aprendizaje, y el 92.23\% considera que permite asociar las características del pensamiento computacional y por ello se debe fortalecer la configuración que actualmente posee.

Se aprecia que los maestrantes consideran al GBCD como complemento de suma importancia para la investigación aplicada, además de ser una herramienta de sostén que permite comprender un saber especifico, facilita el proceso formativo y promueve la autonomía. Esos atributos emergen gracias a las actividades y recursos empleados en el desarrollo de los módulos, razón por la que el gestor se convierte en un mecanismo mediante el cual se pueden lograr nuevas maneras de enseñar o de aprender. En cierta medida, lo antedicho se ajusta a lo expuesto por Lamas et al. (2010, citados por Inzunza et al., 2012) "en cuanto a que permite la flexibilización del tiempo y del espacio, poder contar con una amplitud de recursos, trabajar independiente del horario de clase, la organización que se hace de la asignatura y facilitarles el estudio en su hogar". 
Tabla 3. Resultados del grado de satisfacción del maestrante con el gestor bibliográfico

\begin{tabular}{|c|c|c|c|c|}
\hline Indicador & $\begin{array}{l}\text { Muy de } \\
\text { acuerdo }\end{array}$ & Acuerdo & Indiferente & Desacuerdo \\
\hline $\begin{array}{l}\text { El gestor bibliográfico es importante para mí proceso formativo e } \\
\text { investigativo }\end{array}$ & 45 & 51 & 2 & 5 \\
\hline $\begin{array}{l}\text { La información del gestor bibliográfico permite obtener mejores } \\
\text { resultados para mi investigación }\end{array}$ & 43 & 52 & 4 & 4 \\
\hline $\begin{array}{l}\text { La información del gestor bibliográfico permite hacer feedback } \\
\text { investigativo o evaluativo }\end{array}$ & 30 & 61 & 9 & 3 \\
\hline $\begin{array}{l}\text { La información del gestor bibliográfico permite resolver dudas } \\
\text { académicas o investigativas }\end{array}$ & 33 & 58 & 8 & 4 \\
\hline $\begin{array}{l}\text { El gestor bibliográfico colaborativo permite asociar las características del } \\
\text { pensamiento computacional }\end{array}$ & 42 & 53 & 2 & 6 \\
\hline $\begin{array}{l}\text { La estructura del gestor bibliográfico ayuda a conocer el estado del arte } \\
\text { de un objeto de estudio }\end{array}$ & 34 & 62 & 3 & 4 \\
\hline El gestor bibliográfico posibilita el trabajo autónomo & 34 & 60 & 5 & 4 \\
\hline El gestor bibliográfico posibilita el trabajo colaborativo. & 43 & 49 & 6 & 5 \\
\hline $\begin{array}{l}\text { El gestor bibliográfico colaborativo ayuda a integrar conceptos de } \\
\text { diversas áreas del saber }\end{array}$ & 40 & 55 & 4 & 4 \\
\hline $\begin{array}{l}\text { El gestor bibliográfico dilucida conceptos de interés para su posterior } \\
\text { aplicación }\end{array}$ & 35 & 57 & 7 & 4 \\
\hline
\end{tabular}

Fuente: elaboración propia. 


\section{Gráfica 2. Grado de satisfacción del maestrante con el GBCD}

El gestor bibliográfico dilucida conceptos de interés para su posterior aplicación

El gestor bibliográfico colaborativo ayuda a integrar conceptos de diversas áreas del saber

El gestor bibliográfico posibilita el trabajo colaborativo

El gestor bibliográfico posibilita el trabajo autónomo

La estructura del gestor bibliográfico ayuda a conocer el estado del arte de un objeto de estudio

El gestor bibliografía colaborativo permite asociar las caracterísiticas del pensamiento computacional

La información del gester bibliográfico permite resolver dudas académicas o investigativas

La información del gestor bibliográfico permite hacer feedback investigativo o evolutivo

La información del gestor bibliográfico permite obtener mejores resultados para mi investigación

El gestor bibliográfico es importante para mi proceso formativo e investigativo

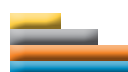

표
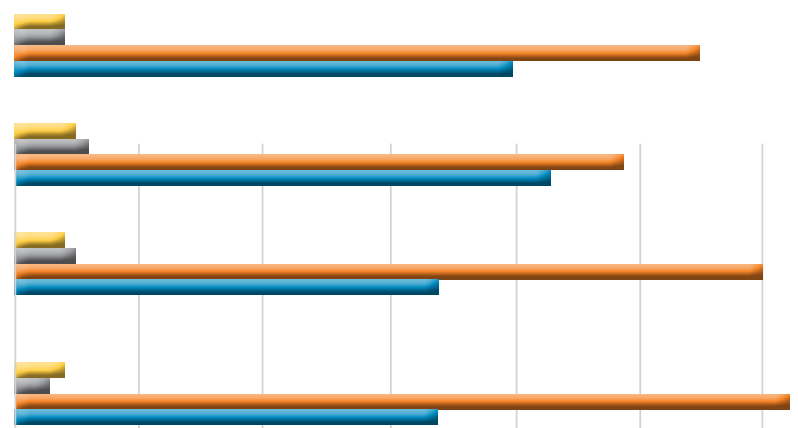



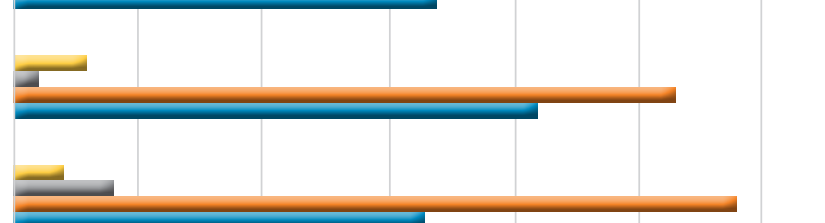

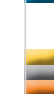
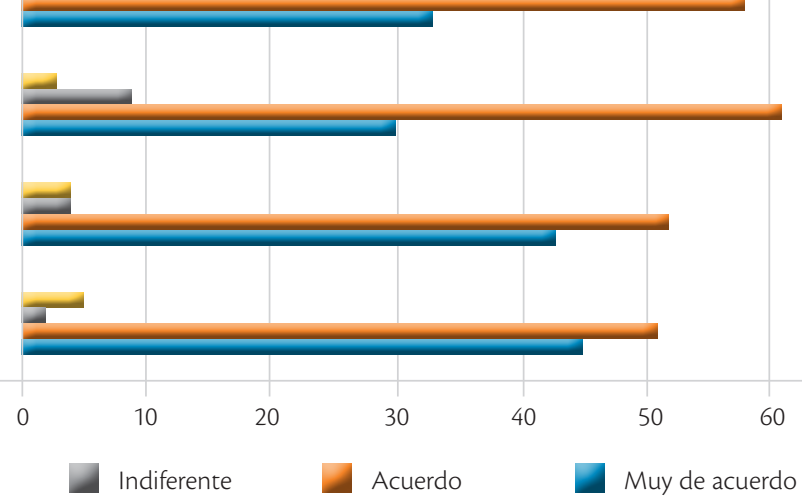

Mediante el gestor se logró generar un escenario donde el estudiante investigador pudo abordar contenidos sobre su objeto de estudio y valuar su nivel de discurso descriptivo, explicativo o argumentativo, de ahí que se llevaron a cabo recursos de valor como la gestión de fichas bibliográficas, análisis y procesos autoevaluativos que, de un modo u otro, contribuyen al desarrollo de las distintas fases de la investigación. De los indicadores expuestos en la tabla 2, el mejor evaluado corresponde a: "el gestor bibliográfico es importante para mí proceso formativo e investigativo" $(93.20 \%)$, porque es muy didáctico, con una configuración clara y puede ser complementado con otros mecanismos de participación como el foro virtual y la socialización de infografias; el 93.20\% de los

maestrantes tienen la certeza de que la estructura del gestor bibliográfico ayuda a conocer el estado del arte de un objeto de estudio y el $92.23 \%$ cree que permite integrar conceptos de diversas áreas del saber.

Desde sus respuestas, los educandos consideran que el gestor bibliográfico posibilita el trabajo autónomo (91.26\%), lo cual es favorable, ya que ello les permite organizar sus tiempos y prepararse mejor a medida que alimenta su capital cultural y participan proactivamente en el entorno virtual de aprendizaje. De la misma manera, los actores del proceso formativo perciben que la información del gestor bibliográfico permite obtener mejores resultados para el proceso investigativo $(93.20 \%$ ), por eso, se puede decir que de una forma u otra el GBCD contribuye 
a que estos aprendan de manera clara y sencilla. Por tanto, la condición no obligatoria respecto al desarrollo de actividades complementarias, es de gran importancia para fortalecer el aprendizaje y la investigación.

También se encontró que los educandos (88.34\%) tienen la certeza de que la información propiciada por el gestor bibliográfico permite resolver dudas académicas o investigativas. A su vez, creen que es necesario ahondar más en discusiones, reflexiones y toma de decisiones desde procesos de interacción social, donde la herramienta tecnológica sea de acceso abierto, incorpore nuevos canales de comunicación y actúe como mediadora a medida que el maestrante se responsabiliza de su aprendizaje. Del mismo modo, se percibe que para los participantes del presente estudio, el gestor bibliográfico dilucida conceptos de interés para su posterior aplicación (89.32\%), de ahí que termina impactando positivamente en la autonomía, el e-Learning, el trabajo interactivo, al igual que en la gestión de la información y del conocimiento.

Finalmente, los hallazgos animan a seguir investigando y replicando la importancia del trabajo multidisciplinario a través del GBCD, ya que permiten potenciar competencias tecnológicas, investigativas y disciplinares, así como desmoronar límites de tiempo y espacio que en ocasiones predominan o pueden ser impuestos indirectamente desde escenarios virtuales de aprendizaje. Si se quiere seguir acrecentando la productividad académica y científica, los esfuerzos deben centrarse principalmente en optimizar la gestión tecnológica y del conocimiento.

\section{Conclusiones}

Las TIC han sido importantes para la sociedad del conocimiento, de ahí que seleccionarlas, valorarlas e implantarlas en la gestión educativa para satisfacer necesidades y problemáticas relacionadas con la formación virtual, la investigación científica y la didactización tecnológica, representa un reto para dependencias y actores del proceso educacional.

Los resultados expuestos permiten inferir que el gestor bibliográfico digital colaborativo se convierte en una estrategia que ayuda en la exploración, clasificación y análisis de fichas bibliográficas para el desarrollo de actividades académicas y de estudios, que de una forma u otra propician mejores resultados de consultas e indagaciones; las competencias tecnológicas logradas pueden fortalecer el $e$-Learning y cualificar la calidad investigativa. Los resultados obtenidos dejan ver un alto grado de aceptación y utilización de dicho gestor, dilucidando la eficiencia y eficacia del dispositivo tecnológico en mención en el desarrollo de los módulos de Caracterización de la investigación aplicada y Propuesta de investigación aplicada.

Además de lo anterior, los estudiantes develan una actitud positiva al momento de trabajar en entornos virtuales de aprendizaje con herramientas TIC que sean pertinentes y lleven a un aprendizaje significativo; el gestor empleado para el desarrollo de los módulos ya mencionados, fortalece el proceso formativo e investigativo, al igual que el trabajo colaborativo; la estructura del artefacto tecnológico incidió satisfactoriamente en el desarrollo de tareas específicas de indagación y, a su vez, posibilitó que el maestrante incorporase sus conocimientos previos o experiencias extracurriculares en beneficio académico y pedagógico; por último, los contenidos que posee el gestor bibliográfico en estudio auspiciaron la comprensión de los objetos de estudio, el rendimiento académico, la capacidad de aprender tanto de manera autónoma como colaborativa y el desarrollo de competencias comunicativas y tecnológicas.

Finalmente, la universidad asume el compromiso de formar maestrantes con un perfil profesional y ocupacional que el ámbito investigativo científico y la sociedad requiere, como el de ser íntegro y responder a los desafíos que el entorno inmediato le plantee. 


\section{Referencias}

Armenteros, Ileana y Alfonso Ileana (2004), "Los gestores personales de bases de datos bibliográficas: conoce usted qué es y cómo se maneja el Procite", Acimed, vol. 12, núm. 2, pp. 1.

Bautista, Juan (2007), "Importancia de las TIC en el proceso de enseñanza y aprendizaje", Blog Comunidades Virtuales, <http://comunidadesvirtuales.obolog.com> [Consulta: abril de 2020].

Bertrand, D. y C. R. Bader (1980), "Storage and retrieval of bibliographic references using a microprocessor system", International Fournal of Bio-Medical Computing, vol. 11, núm. 4, pp. 285-293, <sciencedirect.com/ science/article/abs/pii/ $0020710180900331>$ [Consulta: abril de 2020].

Bianco, Carlos, Gustavo Lugones y Fernando Peirano (2016), "Propuesta metodológica para la medición de la Sociedad del Conocimiento en el ámbito de los países de América Latina", Revista Iberoamericana de Ciencia, Tecnología y Sociedad-CTS, núm. 1, pp. 123.

Cárdenas, Mirian, Eduardo Díaz, Karina Plúa y Mariana Solís (2019), Las TIC en la educación superior, paradigma contemporáneo, Guayaquil, Editorial Colloquium.

Casco, Andrea, Luis Álvarez y Raúl Garrido (2019), "Análisis de los costos ocultos en el proceso de producción industrial”, Revista de investigaciones SIGMA, vol. 6, núm. 2, pp. 51-61.

Duarte, Emilio (2007), "Gestores personales de bases de datos de referencias bibliográficas: características y estudio comparativo", El profesional de la información, vol. 16, núm. 6, pp. 647-656.

Fernández, Ana, María Goicochea, Luis Hernández y Dámaso López (2012), Filología y tecnología. Introducción a la escritura, la informática, la información, Madrid, Editorial Complutense.

Fernández, Francisco (2013), "Sobre comercio electrónico en la WEB 2.0 y 3.0", Revista Cubana de Ciencias Informáticas, vol. 7, núm. 3, pp. 96-113.

Góngora, Yisell y Olga Martínez (2012), "Del diseño instruccional al diseño de aprendizaje con aplicación de las tecnologías", Teoría de la Educación. Educación y Cultura en la Sociedad de la Información, vol. 13, núm. 3, pp. 342-360.

Guacaneme, Marisol (2014), "Uso de recursos educativos abiertos (REA) por profesores de primaria", Tesis de maestría, México, Tecnológico de Monterrey.

Guarnieri, Griselda (2018), "Lógicas de inclusión/ exclusión en la sociedad red: las tecnologías como ampliación del espacio público en la educación superior contemporánea", en M. Casarin (comp.), En torno a las ideas de Manuel Castells: discusiones en la era de la información, Córdoba, Centro de Estudios Avanzados.

Inzunza, Bárbara, Ricardo Rocha, Carolina Márquez y María Duk (2012), "Asignatura virtual como herramienta de apoyo en la enseñanza universitaria de ciencias básicas: implementación y satisfacción de los estudiantes", Formación Universitaria, vol. 5, núm. 4, pp. 3-14.

Kunin, Calvin (1985), "Managing bibliographic citations using microcomputers", The American fournal of Medicine, vol. 78, núm. 4, pp. 627-634, <https:// www.sciencedirect.com/science/article/abs / pii/0002934385904061> [Consulta: abril de 2020].

Lamas, María, Ana Massie y Edmundo Quero (2010), "Implementación de un aula virtual bajo la modalidad mixta: el caso de química agrícola en la Universidad Nacional de Salta", Formación Universitaria, vol. 3, núm 4, pp. 3-12, DOI: http://dx.doi.org/10.4067/S071850062010000400002 [Consulta: junio de 2020].

Latorre, Marino (2018), Historia de las web, 1.0, 2.0, 3.0 y 4.0, Universidad Marcelino Champagnat, <umch. edu.pe/arch/hnomarino/74_HistoriadelaWeb.pdf> [Consulta: mayo de 2020].

Litwin, Edith (2005), Tecnologías educativas en tiempos de Internet, Buenos Aires, Editorial Amorrortu.

Martínez, Raidell, Ibrahim Lorenzo, Magaly Castañeda y María Quintana (2013), “Zotero, más allá de un gestor bibliográfico. Una experiencia con los docentes y nuevas metas", DIM: Didáctica, Innovación y Multimedia, núm 25, pp. 1-13. 
Martínez, Jesús (2021), "Tendencias de la investigación educativa en lenguas extranjeras", Revista Varela, vol. 21, núm. 58, pp. 37-44.

Mayan, María (2001), "Una introducción a los métodos cualitativos: módulo de entrenamiento para estudiantes y profesionales (eBook)", International Institute for Qualitative Methodology, vol. 3, núm. 4, pp. 34-46, <http://www.ualberta.ca/ iiqm//pdfs/introduccion. pdf $>$ [Consulta: mayo de 2020].

Méndez, Michely, Oscar Peñaranda y Karen Rojas (2018), "Diseño de una revista digital que visibilice la diversidad cultural de la ciudad de Cartagena de Indias", Tesis doctoral, Cartagena, Universidad de Cartagena.

Mercado, William, Griselda Guarnieri y Guillermo Rodríguez (2019), "Análisis y evaluación de procesos de interactividad en entornos virtuales de aprendizaje", Revista Trilogía Ciencia Tecnología Sociedad, vol. 11, núm. 20, pp. 63-99, DOI: https://doi. org/10.22430/21457778.1213 [Consulta: mayo de 2020].

Olsen, Wendy (2004), "Triangulation in social research: qualitative and quantitative methods can really be mixed", Development in Sociology, vol. 20, pp. 103-118.

Ramdhani, Abdullah, Muhammad Ali Ramdhani y Abdusy Amin (2014), "Writing a literature review research paper: a step-by-step approach", International fournal of Basic and Applied Science, vol. 3, núm 1, pp. 47-56.

Reyes, Juan, Mirian Cárdenas y Ricardo Aguirre (2020),
"Los gestores bibliográficos, una herramienta de apoyo al proceso investigativo en los estudiantes de Agronomía", Universidad y Sociedad, vol. 12, núm. 1, pp. 232-236.

Rincón, Carlos, Diana Hermith y Wilson Bautista (2018), "Innovación social y su importancia en la gestión del conocimiento y la participación ciudadana", Revista Trilogía Ciencia Tecnología Sociedad, vol. 10, núm. 18, pp. 51-61.

Rodríguez, Kevin (2020), "Desarrollo de un envoltorio del api-rest de mendeley con Graphql", Bachelor's thesis, <http://repositorio.utn.edu.ec/handle/123456789/ 10292> [Consulta: junio de 2020].

Rodríguez, Óscar (2005), "La triangulación como estrategia de investigación en ciencias sociales", Revista Madrid, vol. 31, núm. 2, <https://dialnet.unirioja.es/ servlet/articulo? codigo $=1284197>$ [Consulta: junio de 2020].

Rost, Alejandro (2004), "Pero, ¿de qué hablamos cuando hablamos de interactividad?", La Plata, Congresos ALAIC/IBERCOM 2004, <https://es.scribd.com/ document/35884178/Rost-2004-Pero-de-que-hablamoscuandointeractividad $>$ [Consulta: junio de 2020].

Sandín, María (2003), Investigación cualitativa en educación. Fundamentos y tradiciones, Madrid, Editorial McGrawHill.

Solórzano, Cristian (2016), "Informe de trabajo de investigación de la supervisión 169", Revista digital d'humanitats, núm. 1.

\section{Cómo citar este artículo:}

Mercado-Borja, William-Enrique, Juancri-Rusbel Barrera-Navarro y Roisman-Enrique Ravelo-Ménde (2022), "El gestor bibliográfico digital colaborativo como herramienta de apoyo al proceso investigativo", Revista Iberoamericana de Educación Superior (RIES), vol. XIII, núm. 36, pp. 201-215, DOI: https://doi.org/10.22201/iisue.20072872e.2022.36.1191 [Consulta: fecha de última consulta]. 